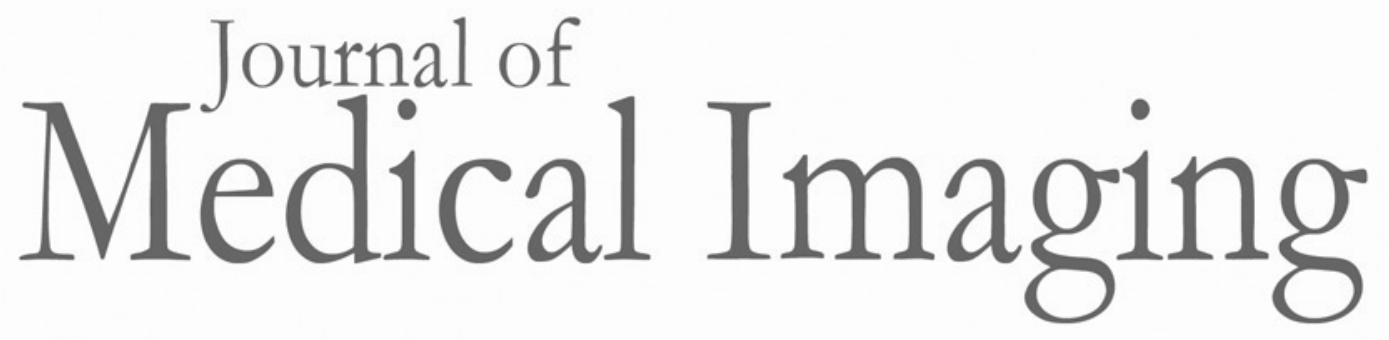

Medicallmaging.SPIEDigitalLibrary.org

\title{
Active monitoring of formaldehyde diffusion into histological tissues with digital acoustic interferometry
}

Daniel R. Bauer

Benjamin Stevens

David Chafin

Abbey P. Theiss

Michael Otter

\section{SPIE.}




\title{
Active monitoring of formaldehyde diffusion into histological tissues with digital acoustic interferometry
}

\author{
Daniel R. Bauer, ${ }^{*}$ Benjamin Stevens, David Chafin, Abbey P. Theiss, and Michael Otter
}

Ventana Medical Systems Inc., 1910 East Innovation Park Drive, Tucson, Arizona 85755, United States

\begin{abstract}
The preservation of certain labile cancer biomarkers with formaldehyde-based fixatives can be considerably affected by preanalytical factors such as quality of fixation. Currently, there are no technologies capable of quantifying a fixative's concentration or the formation of cross-links in tissue specimens. This work examined the ability to detect formalin diffusion into a histological specimen in real time. As formaldehyde passively diffused into tissue, an ultrasound time-of-flight (TOF) shift of several nanoseconds was generated due to the distinct sound velocities of formalin and exchangeable fluid within the tissue. This signal was resolved with a developed digital acoustic interferometry algorithm, which compared the phase differential between signals and computed the absolute TOF with subnanosecond precision. The TOF was measured repeatedly across the tissue sample for several hours until diffusive equilibrium was realized. The change in TOF from 6-mm thick ex vivo human tonsil fit a single-exponential decay $\left(R_{\text {adj }}^{2} \geq 0.98\right)$ with rate constants that varied drastically spatially between 2 and $10 \mathrm{~h}(\sigma=2.9 \mathrm{~h})$ due to substantial heterogeneity. This technology may prove essential to personalized cancer diagnostics by documenting and tracking biospecimen preanalytical fixation, guaranteeing their suitability for diagnostic assays, and speeding the workflow in clinical histopathology laboratories. $\odot$ The Authors. Published by SPIE under a Creative Commons Attribution 3.0 Unported License. Distribution or reproduction of this work in whole or in part requires full attribution of the original publication, including its DOI. [DOI: 10.1117/1.JMI.3.1.017002]
\end{abstract}

Keywords: ultrasound imaging; preanalytical tissue processing; cancer biomarker detection; time of flight; phosphoprotein preservation.

Paper 15217PR received Nov. 12, 2015; accepted for publication Jan. 6, 2016; published online Feb. 8, 2016.

\section{Introduction}

Histological staining of tissue samples obtained from resections or biopsies is the gold standard for detecting and identifying cancer or disease states. Modern histology techniques are built around the concept of fixing tissues with chemicals that cross-link the biostructure of the tissue. This process inhibits metabolism to prevent specimen degradation and preserves biomolecules and tissue structure. ${ }^{1,2}$ The most commonly used fixative in clinical settings is $10 \%$ neutral buffered formalin (NBF), which is an aqueous solution of $3.7 \% \mathrm{w} / \mathrm{v}$ formaldehyde in a buffer. ${ }^{3}$ Currently, histology laboratories process tissue samples in NBF for various periods of time ranging from hours to several days. ${ }^{4}$ In these unstandardized methods, sample quality is empirically determined by a pathologist who examines hematoxylin and eosin (H\&E) stains under a microscope for manifestations of improper fixation, although no quantitative or objective data about fixation quality is available. This subjectivity and lack of quality data is particularly worrisome considering that the majority of mistakes with clinical samples (up to twothirds) occur during the preanalytical phase, meaning that significant errors in diagnosis are potentially being made because of our current inability to detect faulty fixation. ${ }^{5,6} \mathrm{New}$ technologies are therefore needed to monitor and optimize tissue fixation in real time so that each tissue sample is properly fixed

*Address all correspondence to: Daniel R. Bauer, E-mail: daniel.bauer.db2@ roche.com and the most accurate diagnosis is delivered from every sample, from every patient, every time.

Most fixation protocols involving formalin employ the fixative at room temperature, although these protocols can have durations spanning from several hours to days depending on a tissue's thickness, the types of tissue present within a sample, and preanalytical variables such as reagent purity. As demand intensifies for reduced turnaround times, more rapid protocols have been introduced. ${ }^{7-9}$ One such technology is simply to raise the temperature of the fixative to increase the cross-linking rate. While this approach can reduce turnaround time, the use of elevated temperatures alone has led to many reports of unsatisfactory tissue morphology and variability in other molecular assays, including routine immunohistochemistry (IHC) stains. ${ }^{9}$ Alternatively, processing with microwaves has exhibited progress recently, but the technique can cause uneven fixation and tissue damage. ${ }^{10,11}$ Another recent method reported to have superior tissue fixation qualities first incubates tissue in cold NBF, followed by a short incubation in warm NBF. The cold step suppresses enzymatic actions associated with analyte degradation while simultaneously facilitating diffusion of formaldehyde throughout the tissue, and the ensuing warm step rapidly forms formaldehyde linkages to complete the fixation process. ${ }^{12,13}$ Because sparse cross-linking takes place at cold temperatures, this method absolutely requires sufficient concentrations of formaldehyde to have diffused into the tissue so that the subsequent warm step does not simply heat tissue in the absence of fixative. This preservation method was shown to preserve protein epitopes with notorious preanalytical sensitivity, 
such as phosphorylated protein kinase B (pAKT) and phosphorylated epidermal growth factor receptor, which are not currently clinically evaluated but are known to be key biomarkers associated with several forms of cancer. ${ }^{14-18}$ Additionally, clinically assessed biomarkers like estrogen receptor, Ki-67, hormone receptor, and human epidermal growth factor receptor 2 are also known to be sensitive to improper fixation, indicating a broad applicability of this technique. ${ }^{19-21}$

At present, there are no technologies capable of quantifying the concentration of formaldehyde or the subsequent formation of cross-links in tissue specimens. Diffusion of an exogenous chemical into a tissue is a complicated process that is markedly influenced by temperature, tissue heterogeneity, the molecular size and shape of the penetrating chemical, and the specific type and relative composition of the tissue sample. ${ }^{22}$ Some researchers have soaked tissues in radioactive formaldehyde and used photography film as a measure of diffusion rates. ${ }^{23,24}$ However, long exposure times and low radioactivity actually incorporated into the tissue generate confusing and unreliable results, and a radiologic technique would be incompatible with routine application in the clinical laboratory. Others have used ultrasound (US) detection to investigate cross-linking by comparing the acoustic properties of samples before and after room temperature fixation. ${ }^{25}$ Ultimately, neither of these techniques would enable real-time monitoring when changes could be implemented to guarantee proper tissue fixation and ideal biomarker preservation. For these reasons, we sought to develop a real-time method of monitoring formaldehyde diffusion into tissue.

We report here the development of a semiautomated realtime diffusion monitoring technology based on measuring a change in the speed of US waves through ex vivo tissues. ${ }^{26}$ Time-of-flight (TOF) measurements were acquired as formaldehyde passively diffused into tissues and changed their physical composition in a way that altered the overall acoustic transit time. The US thus accumulated a transit time differential proportional to the amount of fluid exchange (i.e., the formaldehyde concentration), which was resolved with digital acoustic interferometry with subnanosecond sensitivity. With this technology, we were able to track and quantify formaldehyde diffusion dynamically until the tissue and bulk fixative became isotonic, resulting in maximum formaldehyde concentration in the tissue. Ultimately, this technology could be integrated into commercial tissue processors to standardize and optimize tissue fixation and thereby speed the diagnosis and classification of pathologic processes in tissue by rapidly preserving critical yet labile biomarkers.

\section{Methods and Materials}

\subsection{Theory}

Pairs of $4 \mathrm{MHz}$ focused transducers were spatially aligned and a sample was placed close to their common foci. One transducer, designated the transmitter, sent out an acoustic pulse that traversed both the coupling fluid, which was typically formalin, and the tissue sample before being detected by the receiving transducer. The TOF through only the formalin (reference channel) was subtracted from the TOF with the tissue sample present to isolate the phase retardation from the tissue and to compensate for environmentally induced fluctuations in the reagent (Fig. 1). This process was repeated to detect the changing transit time through the tissue during passive diffusion.

Due to the distinct sound velocities of formalin and exchangeable fluid within the sample, as formaldehyde diffused into the tissue, the overall transit time was slightly altered. The form of the change in TOF from the tissue $\left(\Delta \mathrm{TOF}_{\text {tissue }}\right)$ can be written

$$
\Delta \mathrm{TOF}_{\text {tissue }}(t)=\frac{D}{r_{o}+\rho \cdot\left(r_{\text {bulk }}-r_{o}\right) \int_{0}^{D} c(t, r) \mathrm{d} r},
$$

where $D$ is the tissue's diameter, $\rho$ is the tissue's porosity, $r_{\text {bulk }}$ is the sound velocity of the bulk reagent, $r_{o}$ is the sound velocity of the undiffused tissue, and $c$ is the concentration of the exogenous cross-linking agent, which varies in time $(t)$ and space $(r)$. The change in the speed of sound was scaled by the porosity of the tissue, which varied between 0 and 1 and represents the volume fraction of the tissue that was eligible for diffusion. Equation (1) models the speed of sound in the tissue as a linear combination of the tissue's original sound velocity and the sound velocity of the bulk fluid. The detected change in TOF will thus be inversely proportional to the speed of sound differential of the two fluids. As an example, an US pulse will take $2666.6 \mathrm{~ns}$ to traverse a 4-mm specimen whose sound velocity is $1500 \mathrm{~m} / \mathrm{s}$. Assuming that the bulk media's sound velocity was $10 \%$ higher and the tissue had a porosity of $10 \%$, after reaching equilibrium, an US pulse will take $2640.5 \mathrm{~ns}$ to traverse the tissue, generating $26 \mathrm{~ns}$ of TOF differential when osmotic equilibrium is achieved. Equation (1) thus predicts diffusion will change the acoustic transit time across a tissue on the order of tens of nanoseconds.

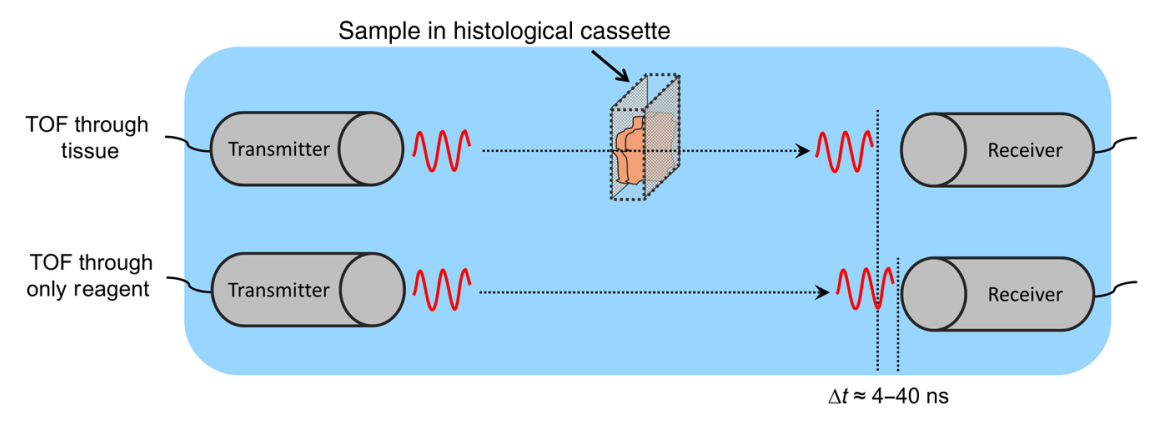

Fig. 1 Schematic representation of the acoustic TOF diffusion monitoring system. Transit times of acoustic pulses traversing the formalin and tissue (top row) and a reference acquisition through only the formalin (bottom row) were calculated. Reference channel subtraction eliminated environmentally induced noise and isolated the TOF contribution from the tissue. 


\subsection{Experimental Setup}

A commercial dip and dunk tissue processor (Lynx II, Electron Microscopy Sciences) was retrofitted with custom acoustic hardware; see Fig. 2(a). A mechanical head was designed in Solidworks ${ }^{\circledR}$ to fit around and seal a standard Lynx II reagent canister. A cassette holder was designed for use with a biopsy compatible cassette (Leica Biosystems, CellSafe Biopsy Capsules) to securely hold smaller tissue samples (dia. $\leq 4 \mathrm{~mm}$ ) and prevent them from moving. Alternatively, a separate holder was designed for a standard-sized cassette for larger specimens up to $7 \mathrm{~mm}$ thick. The cassette holder held the tissue perpendicular to the propagation axis of the US. The cassette holder was attached to a vertical translation arm that enabled the tissue to be spatially mapped by changing where acoustic beams traversed the tissue sample. Two metal brackets on either side of the tissue cassette [Fig. 2(b)] housed the transmitting and receiving transducers [CNIRHurricane Tech (Shenzhen) Co., $f=12 \mathrm{~mm}, \mathrm{FWHM}=2.2 \mathrm{~mm}]$. The receiving bracket also held a pair of transducers oriented orthogonally to the other transducers [see Fig. 2(c)] to measure only the bulk fluid. These transducers served as a reference channel to compensate for temperature fluctuations in the bulk fluid.

A two-dimensional (2-D) scan was completed by calculating the TOF between all five transducer pairs. The cassette was then translated $\sim 1 \mathrm{~mm}$ vertically and TOF values were calculated at the new position. This process was repeated with 13 or 21 vertical acquisitions for the biopsy and standard-sized tissue cassettes, respectively. The lateral locations of TOF acquisitions from all transducer transmitted-received pairs are displayed in Fig. 2(d). After each translation, the orthogonal reference sensors measured the TOF value through the bulk fluid to account for thermal fluctuations. Additionally, at the end of a 2-D acquisition, the cassette was raised up out of the path of the transducers and a second reference acquisition was acquired. These TOF values were used to detect spatiotemporal variations in the fluid. The process was repeated over the course of the experiment until the tissue reached equilibrium. Acquisition of a 2-D TOF dataset required about $100 \mathrm{~s}$, although without spatial scanning, TOF data could be acquired at $3 \mathrm{~Hz}$.

\subsection{Data Acquisition and Time-of-Flight Calculation Algorithm}

The TOF measurements were calculated using a developed postprocessing algorithm. Initially, the transmitting transducer was set with a programmable waveform generator (AD5930, Analog Devices) to transmit a sinusoidal signal with a frequency of 3.7 MHz for $600 \mu \mathrm{s}$. That pulse was detected by the receiving transducer after traversing the fluid and tissue [Fig. 3(a)]. The received and transmitted sinusoids were compared electronically with a digital phase comparator (AD8302, Analog Devices). The phase comparator had an integration time of $165 \mu \mathrm{s}$ and thus required the transmitted and received pulses to temporally coexist for at least this long to determine the phase relationship between the two signals. After the output of the phase comparator stabilized, it was queried 100 times with an 8-bit analog-to-digital converter (AT91SAM, Atmel), and the average was recorded. The output voltage from the phase comparator had a standard deviation of less than $2 \mathrm{mV}$, which was significantly less than the bit depth. In this manner, the phase relationship between transmitted and received signals was calculated for a sinusoidal signal with a frequency of $3.7 \mathrm{MHz}$. This process was continually repeated by increasing the frequency of the transmitted sinusoidal signal and again recording the phase relationship between the transmitted and received pulses. The phase relationship was calculated for acoustic signals with frequencies ranging from 3.7 to 4.3 MHz with a discrete phase value recorded every $600 \mathrm{~Hz}$ (e.g., $\nu=3.7,3.7006,3.7012 \ldots 4.3 \mathrm{MHz}$ ). This range was chosen because the output of the transducers was sufficiently large between 3.7 and 4.3 MHz. The output of this recording, referred to as a phase-frequency sweep, repeated itself every time the accumulated phase differential completed a cycle. An experimentally acquired phase-frequency sweep is displayed in Fig. 3(b), where 0 volts corresponds to a phase difference of $\pm \pi$ radians and 1.8 volts corresponds to 0 radians.

The voltage from the phase comparator was converted to a temporal phase shift, referred to as the experimental phase $\left(\varphi_{\text {exp }}\right)$. Next, a brute-force simulation was used to calculate phase-frequency sweeps for different TOF values. Candidate temporal phase values, as a function of input sinusoid frequency, were calculated according to (a)

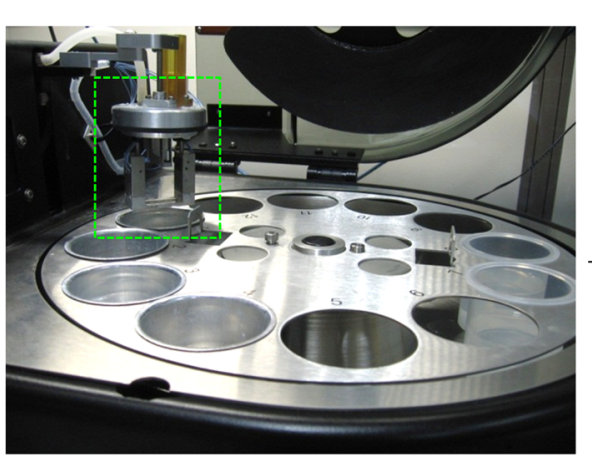

(b)

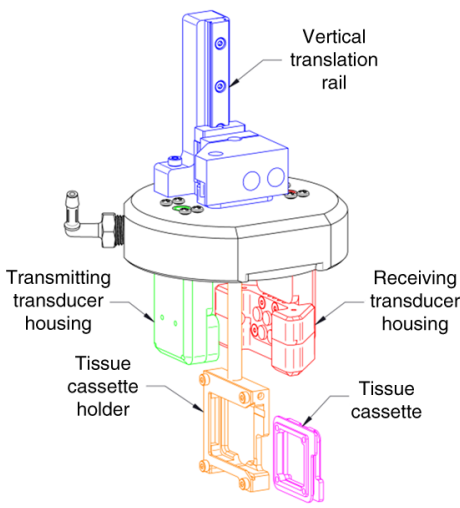

(c)

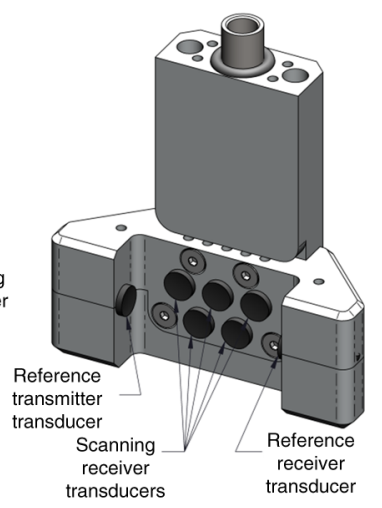

(d)

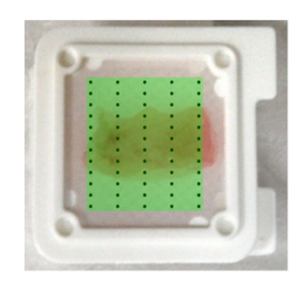

Fig. 2 (a) Lynx II tissue processor modified with US scanning technology as indicated with dashed green box. (b) Solidworks ${ }^{\circledR}$ drawing of scan head with pairs of 4-MHz transducers spatially aligned on either side of the green histological cassette, which was vertically translated to acquire 2-D information. (c) Solidworks ${ }^{\circledR}$ depiction of the receiving fixture. The orthogonal transducer pair served as a reference channel to detect gradients in the bulk reagent. (d) Photograph of biopsy tissue cassette with scanned area indicated with green rectangle and lateral locations of TOF acquisitions indicated with black dots. 
(a)

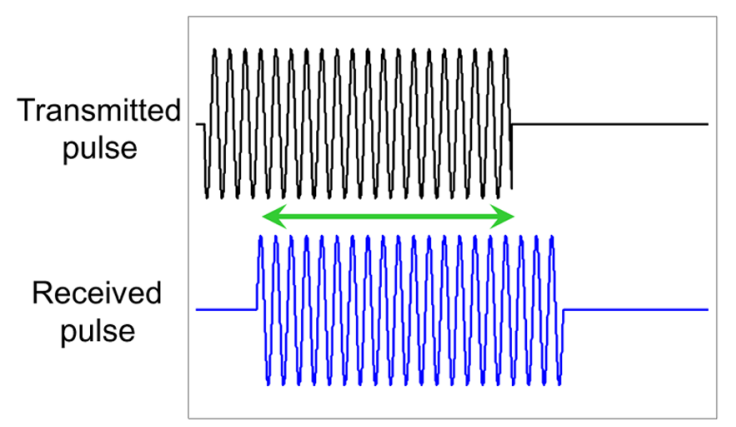

Time axis (b)

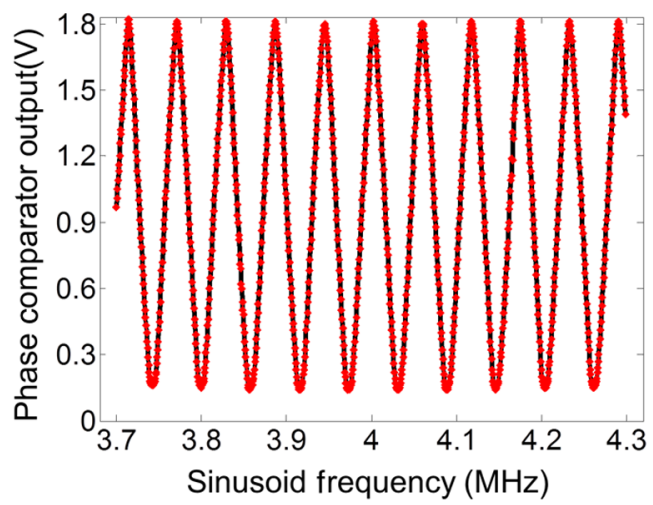

Fig. 3 (a) Qualitative depiction of transmitted and detected acoustic pulses. (b) Output voltage of phase comparator versus frequency of the transmitted sinusoid representing the phase relationship between transmitted and received signals. Values generated during region of overlap (green arrow). Red stars are recorded data points $(v=3.7$ to $4.3 \mathrm{MHz}, \Delta v=600 \mathrm{~Hz}$ ). $0 \mathrm{~V}= \pm \pi \mathrm{rad}, 1.8 \mathrm{~V}=0 \mathrm{rad}$.

$$
\phi_{\text {cand }}\left(\mathrm{TOF}_{\text {cand }}, \nu\right)=\left|\mathrm{TOF}_{\text {cand }}-\operatorname{rnd}\left[\frac{\mathrm{TOF}_{\text {cand }}}{T(\nu)}\right] T(\nu)\right| \text {, }
$$

where $\mathrm{TOF}_{\text {cand }}$ is the candidate TOF in nanoseconds, $T$ is the period of the input sinusoid in nanoseconds, rnd is the "round to the nearest integer" function, and $|\ldots|$ is the absolute value operation. For a given candidate TOF and frequency value (i. e., period), the term on the right represents how long it takes for the nearest number of cycles to occur. This value was subtracted from $\mathrm{TOF}_{\text {cand }}$ to calculate the temporal phase, into or up to the next complete cycle. Phase values were thus computed for multiple candidate TOF values initially ranging from 10 to $30 \mu$ s with 200-ps spacing. The error between experimental and candidate frequency sweeps was calculated in a leastsquares sense for individual candidate TOF values by

Error $\left(\mathrm{TOF}_{\text {cand }}\right)=\sum_{\nu=1}^{N}\left[\phi_{\text {cand }}\left(\mathrm{TOF}_{\text {cand }}, \nu\right)-\phi_{\exp }(\nu)\right]^{2}$,

where $N$ is the total number of frequencies in the sweep. A normalized error function is displayed in Fig. 4(a) and resembles an optical interferogram. For example, each feature has a width of one acoustic period $(T=1 / 4 \mathrm{MHz}=250 \mathrm{~ns})$. The maximum

(a)

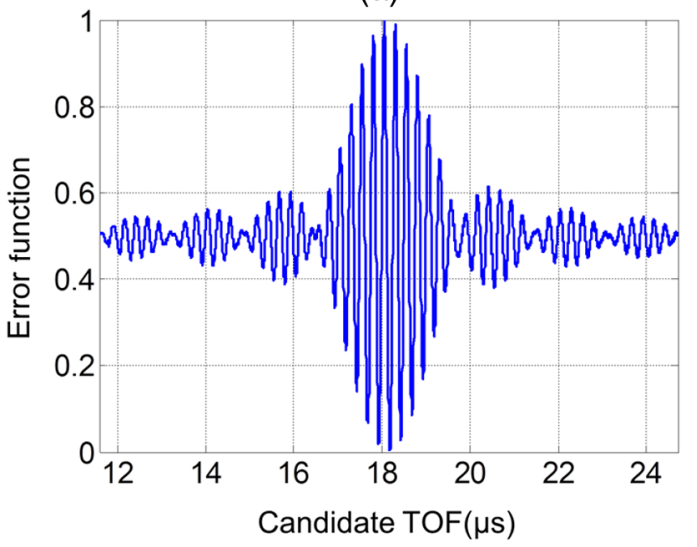

error function indicates the candidate phase-frequency sweep had equal wavelength but was out of phase with the experimental phase-frequency sweep. Conversely, when the error was minimized, the two were completely harmonized, thus the reconstructed TOF is registered as the global minimum of the error function

$\mathrm{TOF}_{\text {recon }}=\underset{\mathrm{TOF}_{\text {cand }}}{\arg \min }($ Error $)$.

The technique of digitally comparing acoustic waves produced high-precision results due to the sharpness of the center trough [Fig. 4(b)]. The error function had a minimum value of 0.0033 at $18175.56 \mathrm{~ns}$, indicating exceptionally well-matched candidate and experimental phase-frequency sweeps. Note that the precision of this method could be increased by filtering the error function, although no signal processing was performed at this step.

\subsection{Environmental Mitigation and Data Processing}

The speed of sound in fluid has a large temperature dependence that is exacerbated because the absolute TOF is an integrated signal over the path length between the transducers. For

(b)

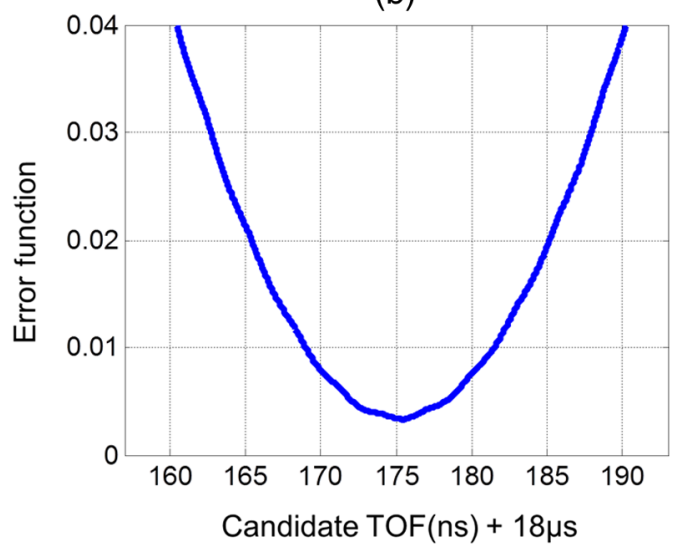

Fig. 4 (a) Normalized error function versus candidate TOF. Function resembles an optical interferogram. (b) Error function near global minimum, which corresponds to the true absolute TOF. 
(a)

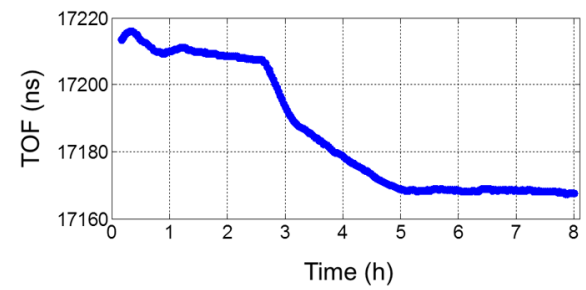

(b)

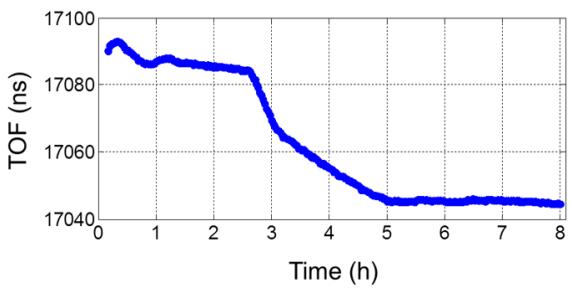

(c)

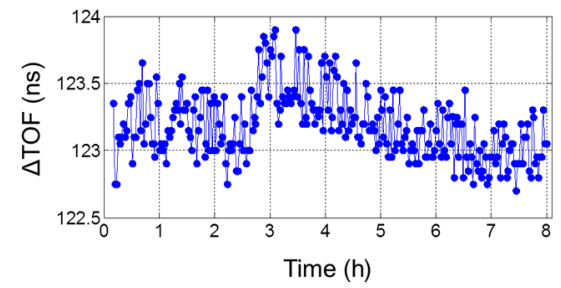

Fig. 5 Absolute TOF between acoustic path lengths traversing (a) an empty cassette and formalin and (b) only the formalin. (c) Reference-compensated TOF calculated as the difference between (a) and (b). Stable reference-compensated TOF demonstrates robust environmental mitigation ( $\sigma=247 \mathrm{ps}$ ).

instance, the time for US to traverse $1 \mathrm{~mm}$ of $4^{\circ} \mathrm{C}$ water will change $2.3 \mathrm{~ns} /{ }^{\circ} \mathrm{C}$. Two mechanisms were employed to mitigate these environmental fluctuations: a proportional-integralderivative (PID) algorithm on the hardware used to cool the bulk fixative solution, and TOF reference compensation through the bulk media. The PID temperature control was based on a pulse width modulation (PWM) algorithm that continually read the temperature of the reagent from a thermistor (Omega TH-1044007), and once per second adjusted how long the cooling hardware was on in $392-\mu$ s increments. The PWM algorithm was found to stabilize the temperature of the fluid with a standard deviation of roughly $0.05^{\circ} \mathrm{C}$ about the set point. Further correction for temperature variance was realized by reading the TOF through only the bulk reagent. This TOF value was subtracted from the TOF through the reagent and tissue to mitigate contributions from environmentally induced fluctuations in the fluid. Best results were achieved with relatively slow low-amplitude transients in the fluid, so the PWM algorithm was programmed to stabilize low-frequency temperature fluctuations while reference compensation eliminated high-frequency variations.

TOF acquisitions with an empty cassette are shown in Fig. 5. The absolute TOF of the formalin slowly changed 40 ns over the 8-h experiment due to thermal drift within the fluid. However, after reference compensation, the change in TOF $(\Delta \mathrm{TOF})$ was essentially flat and only varied $\pm 500 \mathrm{ps}(\sigma=247 \mathrm{ps})$ from its baseline of $123 \mathrm{~ns}$, which was due to the retardation from the plastic mesh in the cassette. Low-order median and smoothing filters, in addition to a third-order Butterworth filter, were used to eliminate stochastic noise while preserving the low-frequency components from the tissue. Filtering typically reduced the noise an additional 25 to $50 \%$.

\subsection{Histologic Imaging and Image Processing}

Calu-3 mouse xenograft tumors were harvested from severe combined immunodeficiency (SCID) mice. Calu-3 is a human airway carcinoma-derived cell line that overexpresses pAKT.

Animals were cared for in accordance with standards established by the International Association for the Assessment and Accreditation of Laboratory Animal Care, and experiments were approved by Roche's Institutional Animal Care and Use Committee. For IHC analyses, tissue samples went through routine processing on a standard tissue processor (Leica ASP300). They were embedded in paraffin, sectioned at $4-\mu \mathrm{m}$ thick, and placed on microscope slides. Samples were stained with an antibody to the phosphorylated form of the AKT protein that is highly fixation sensitive. ${ }^{16,17}$ Imaging of each slide was performed on a microscope (Nikon Eclipse $80 i)$ with a $2 \times$ objective (Nikon Plan Apo). Images from the microscope were compensated for the illumination pattern of the light source by dividing the transmitted image with an a priori flat image acquired with no tissue sample. The intensity image was $\log$ transformed and spectrally unmixed to quantify the relative amount of pAKT at each pixel. A reference tissue stained for pAKT was used to calibrate the color spectrum of staining. A segmentation algorithm was used to identify the tissue border, and the Euclidean distance to the nearest edge pixel was calculated for each pixel within the tissue. Average stain level (in arbitrary units) as a function of distance to the edge was then assessed for each sample versus time in NBF.

\section{Results}

\subsection{Time of Flight from Ex Vivo Tissues}

The TOF system was used to monitor changes within a 6-mm thick human tonsil placed in $6^{\circ} \mathrm{C} 10 \% \mathrm{NBF}$. The reference-compensated TOF from different regions are shown in Fig. 6. The change in TOF displayed a monotonically decreasing signal that was best correlated with a single-exponential function of the form

$$
\Delta \operatorname{TOF}(\vec{r}, t)=C(\vec{r})+A e^{-t / \tau(\vec{r})}
$$

where $C$ is a constant offset in nanoseconds, $A$ is the amplitude of the decay in nanoseconds, $\tau$ is the decay constant in hours, and the spatial dependence $(\vec{r})$ is explicitly stated. The amplitude and decay rate were quantified by fitting to Eq. (5) using nonlinear regression. The TOF signal from the tissue's periphery exhibited a $\triangle \mathrm{TOF}$ change of $8 \mathrm{~ns}$ and reached equilibrium in $\sim 8 \mathrm{~h}$ (top plot). Just $1 \mathrm{~mm}$ toward the center, the $\Delta \mathrm{TOF}$ had a larger decay amplitude of $27 \mathrm{~ns}$ and required nearly $16 \mathrm{~h}$ to reach equilibrium (middle plot). The center of the tissue had the largest amplitude and was still changing after $16 \mathrm{~h}$ (bottom plot). All three signals had adjusted $R^{2}$ values greater than 0.99 and standard errors (SEs) less than 500 ps. The tissue's center would be expected to experience slower diffusion because it had less surface area exposed to the bulk fixative, and increased amplitude because the tissue sample used was thicker toward the middle.

To verify that the change in TOF derived contrast from reagent diffusion, and not from an ancillary effect such as cross-linking, an additional experiment was performed by incubating a sample in differing concentrations of formalin. This experiment exploited the fact the TOF monotonically decreased versus percent formaldehyde [see Fig. 7(a)] to test the reversibility of the TOF signal. The hypothesis of this experiment was that chemical modification (cross-linking) within the tissue would lead to irreversible TOF changes in conditions where cross-links cannot be undone, whereas simple diffusion of 

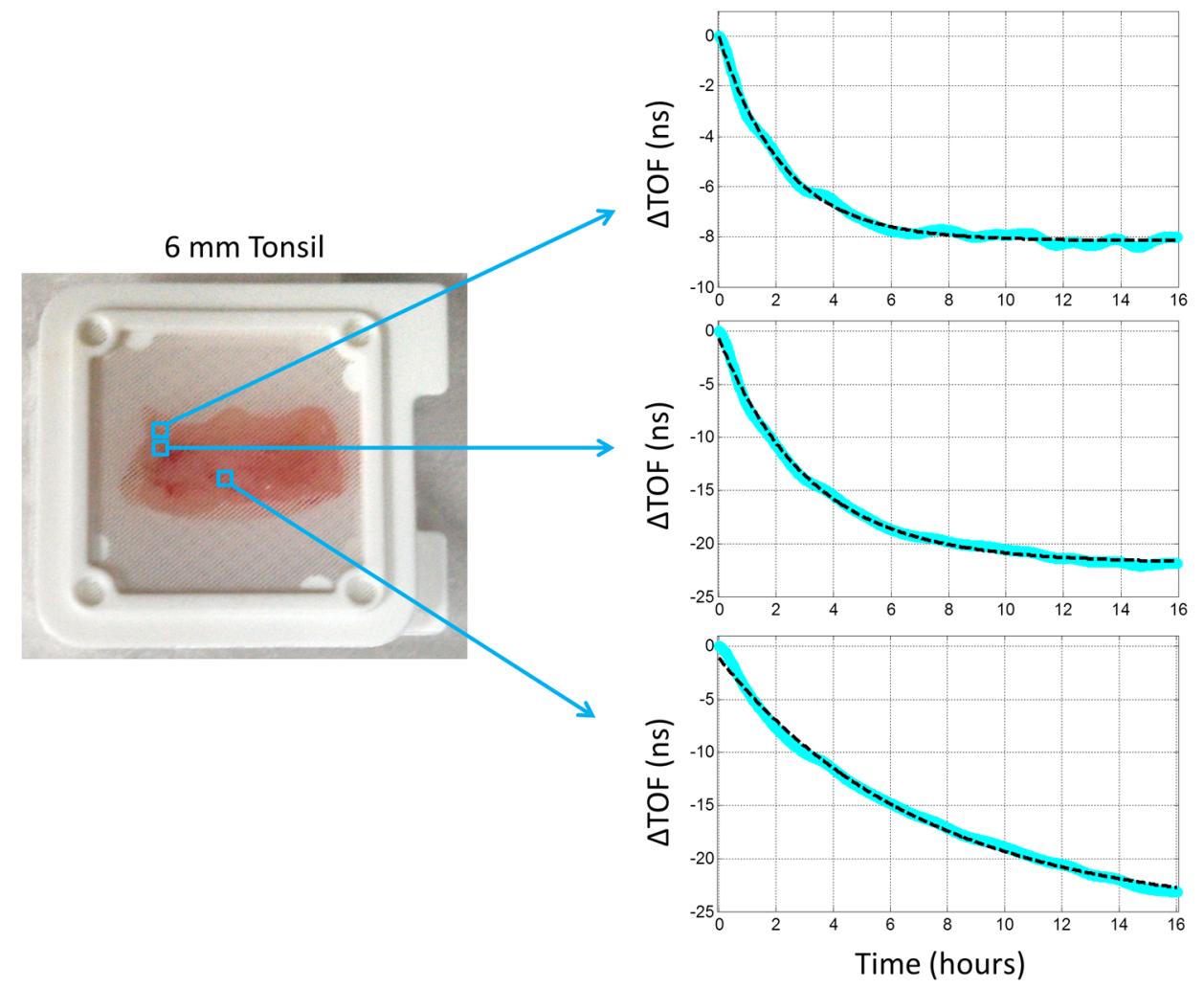

Fig. 6 Measured $\Delta$ TOF from regions in a 6-mm human tonsil completely diffused (top, $R_{\text {adj }}^{2}=0.994$, $\mathrm{SE}=145 \mathrm{ps}$ ), mostly diffused (middle, $R_{\mathrm{adj}}^{2}=0.996, \mathrm{SE}=320 \mathrm{ps}$ ), and partially diffused (bottom, $R_{\mathrm{adj}}^{2}=0.996, \mathrm{SE}=406 \mathrm{ps}$ ) after $16 \mathrm{~h}$ in formalin. All $\Delta \mathrm{TOF}$ offsets were subtracted off.

formaldehyde into and out of tissue would produce TOF changes exhibiting minimal hysteresis. A 4-mm human tonsil was therefore scanned in a series of cold NBF solutions: $10 \%$ formalin, $40 \%$ formalin, $10 \%$ formalin, and finally $40 \%$ formalin over a multiday experiment. The TOF signal, averaged over 12 recorded positions, for each stage is shown in Fig. 7(b). Initially, the $\triangle \mathrm{TOF}$ decreased about $10 \mathrm{~ns}$ as the formalin penetrated into the tissue (see blue curve). An immediate and larger change in the $\triangle \mathrm{TOF}$ was observed when this tissue was placed into $40 \%$ formalin (see magenta curve). Conversely, when the tissue was returned to $10 \%$ formalin, the $\Delta \mathrm{TOF}$ increased (i.e., the speed of sound slowed), but the $\Delta \mathrm{TOF}$ almost entirely reverted to its lower value when returned to $40 \%$ formalin (see red and green curves). The tissue sample at diffusive equilibrium with $10 \%$ and $40 \%$ formalin were 4 to 9 ns and 30 to $37 \mathrm{~ns}$ faster than undiffused tissue, respectively. Additionally, the low spatial variability in each TOF signal ( $\sigma=2$ to $7 \mathrm{~ns})$ provides evidence that the signal was not merely detecting tissue (a)

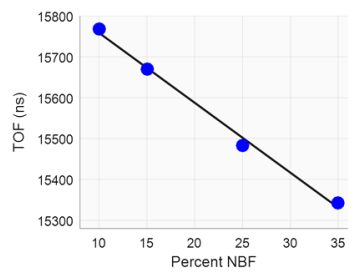

(b)

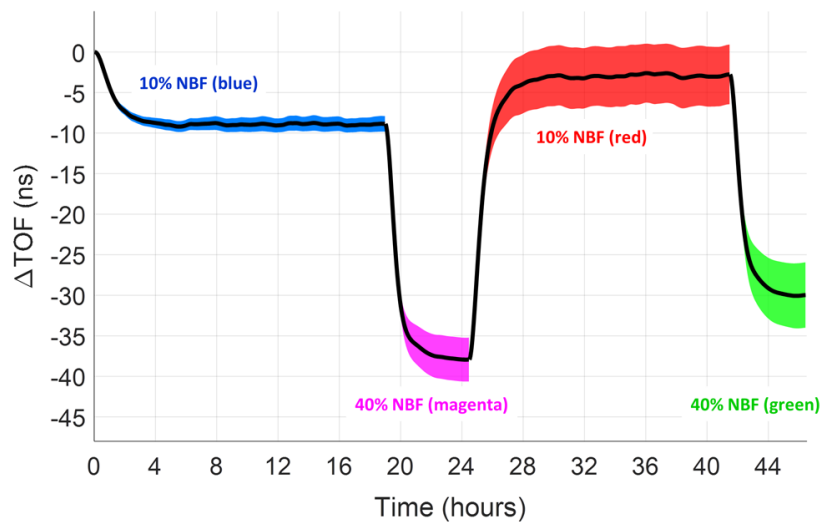

Fig. 7 (a) Measured TOF through $23 \mathrm{~mm}$ of room-temperature fluid, demonstrating that TOF consistently decreased with increasing NBF concentration $\left(R^{2}>0.99\right.$ ). (b) Average $\Delta$ TOF (black line) from 12 different locations in a 4-mm human tonsil placed successively in solutions of $10 \%$ (blue), $40 \%$ (magenta), $10 \%$ (red), and $40 \%$ (green) formalin. Error bar width represents standard deviation. Results elucidate that with a cold fixative, the TOF signal was reversible and consistent with formalin diffusion. $R_{\mathrm{adj}}^{2} \geq 0.99$, $\mathrm{SE} \leq 638 \mathrm{ps}$ for all. 
deformations such as expansions or contractions but rather identifying a continuous physical effect occurring throughout the specimen. The results from this experiment were therefore consistent with diffusion of formaldehyde into or out of the tissue both in terms of the polarity and magnitude of each TOF signal.

\subsection{Quantification of Diffusion Variability}

The TOF system was used to characterize formaldehyde diffusion into tonsil samples 2, 4, and $6 \mathrm{~mm}$ thick [Fig. 8(a)]. As the size of the sample increased, the magnitude of the TOF shift also increased. Decay amplitudes were 5.0, 12.06, and $24.95 \mathrm{~ns}$ for 2, 4, and $6 \mathrm{~mm}$ thick samples, respectively. This was expected because thicker samples had larger volumes of fluid to exchange, resulting in a changed speed of sound over a larger distance, producing shorter cumulative TOF changes. Furthermore, diffusion time also increased dramatically with tissue thickness. The decay constants were $0.55,2.5$, and $6.6 \mathrm{~h}$ for 2-, 4-, and 6-mm samples, respectively. This increase in diffusion time was predicted by Fick's second law, which, to a firstorder approximation, predicts a squared dependence between particle penetration depth and time.

The scanning capability of this technology also enabled probing the spatial variability of formaldehyde concentration changes within the tissue. To examine this, a 6 -mm tonsil biopsy core, $\sim 10 \mathrm{~mm}$ in length, was placed into $6^{\circ} \mathrm{C} 10 \%$ formalin and scanned with the TOF system. Decay constants from the $\Delta$ TOF throughout the sample are displayed in Fig. 8(b). In general, the center portion of the sample had slower diffusion rates (larger decay constants) than the end portions. This is likely because the ends of the tissue had more surface area for active fluid exchange. However, significant differences in the diffusion rates in the middle of the sample were observed (4 to $10 \mathrm{~h}$ ), despite all regions having nearly equal exposed surface area. These large differences in diffusion rates demonstrate how variable fixative diffusion rates can be and why monitoring diffusion could be critical for ensuring successful preservation in all samples.

In a final experiment, our detection system was used to visualize diffusion over time in a 6-mm tonsil. The TOF trends for each spatial location were fit to Eq. (1) and fit amplitudes and decay constants were interpolated to create 2-D mappings of the spatially dependent diffusion process. The time dependence of the diffusion process can be seen in Fig. 9(a), which displays a photograph of the tissue superimposed with contour lines indicating how long regions take to reach $63 \%$ of their maximum formaldehyde concentration. Conversely, Fig. 9(b) depicts a photograph of the tonsil sample overlaid with contour lines labeling what percentage of diffusion had yet to occur after $5 \mathrm{~h}$. A large dependence on distance from the tissue edge was observed because formaldehyde penetrated from the outer surfaces of the tissue toward the interior. However, a large degree of tissue heterogeneity was also observed. For example, the region of tissue at $x=6 \mathrm{~mm}, y=0$ had a decay constant similar to the tissue's center, likely resulting from physical differences in tissue microheterogeneity or thickness.

\subsection{Biomarker Staining Results}

Given that fixatives infiltrate tissue from the edges and outer surfaces, we sought to investigate this effect by observing the preservation of analytes that are especially sensitive to the quality of fixation. ${ }^{16,27,28}$ Calu-3 mouse xenograft tumors were harvested from SCID mice and sliced to no more than $4 \mathrm{~mm}$ thick. Calu-3 is a human airway carcinoma-derived cell line that overexpresses pAKT. Samples were placed in cold formalin, with less than $10 \mathrm{~min}$ of cold ischemia, for 1 to $3 \mathrm{~h}$ before being cross-linked in heated formalin for $2 \mathrm{~h}$. With only 1 or 2 hours of diffusion time in cold formalin, the tissues were almost completely devoid of stain toward their interiors [Fig. 10(a)]. More uniform staining was observed throughout both samples that were subjected to $3 \mathrm{~h}$ of cold formalin, although the center of these samples continued to show less pAKT than was evident at the periphery.

To quantify these subjective observations, image processing was employed to quantify the relative concentration of pAKT versus distance to the nearest edge of the tissue. The staining intensity functions for samples exposed to formalin for 1, 2, and $3 \mathrm{~h}$ are plotted in Fig. 10(b), where the error bar width represents the standard deviation of the two images for each diffusion time. Staining intensity substantially increased with longer diffusion times. Furthermore, augmented stain intensities were observed at all radial edge distances as diffusion time increased, demonstrating that longer diffusion times are necessary to preserve pAKT at the center and periphery of the tissues. On average, samples exposed to formalin for $3 \mathrm{~h}$ preserved $48 \%$ and $117 \%$ more pAKT than 2-h and 1-h samples, respectively [Fig. 10(c)]. We hypothesize that the gradient in pAKT signal was a consequence of inadequate formaldehyde diffusion into the specimen resulting in diminished preservation of this (a)

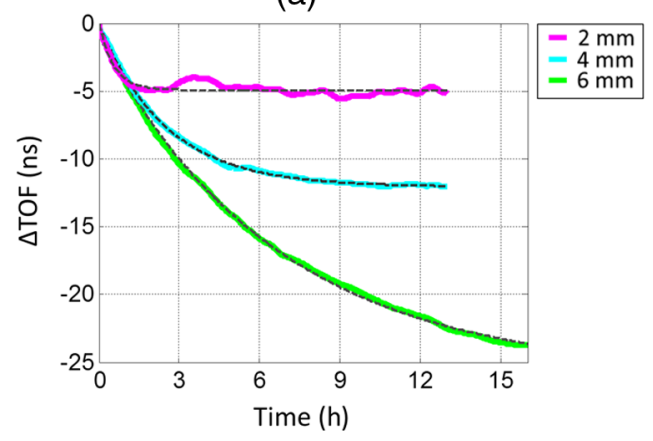

(b)

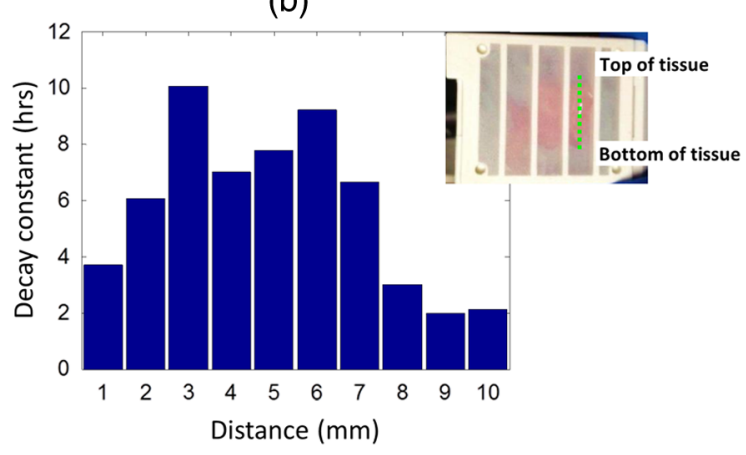

Fig. 8 (a) $\Delta$ TOF from tonsil samples $2 \mathrm{~mm}\left(R_{\mathrm{adj}}^{2}=0.8, \mathrm{SE}=350 \mathrm{ps}\right), 4 \mathrm{~mm}\left(R_{\mathrm{adj}}^{2}=0.999\right.$, SE $\left.=90 \mathrm{ps}\right)$, and $6 \mathrm{~mm}\left(R_{\mathrm{adj}}^{2}=0.998, \mathrm{SE}=230 \mathrm{ps}\right)$ thick in $10 \%$ formalin. All $\Delta$ TOF offsets were subtracted off. (b) Decay constants from 6-mm tonsil, obtained sequentially from top to bottom. Decay constants range from 2 to $10 \mathrm{~h}(\sigma=2.9 \mathrm{~h})$. 
(a)

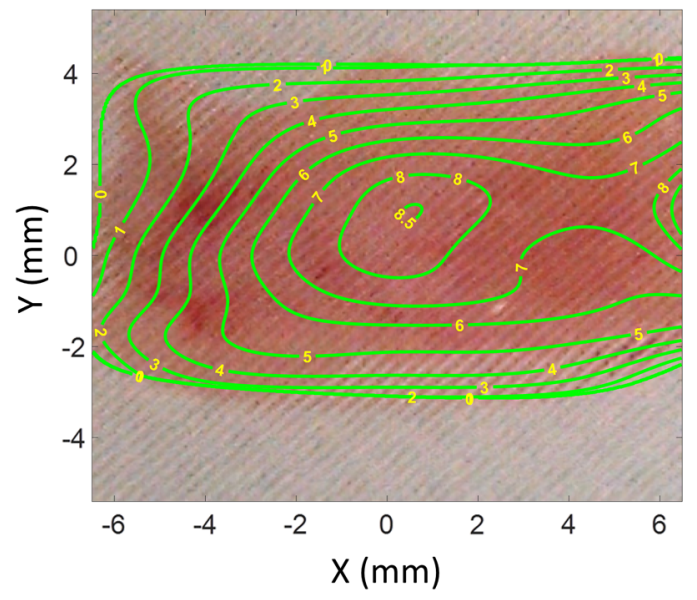

(b)

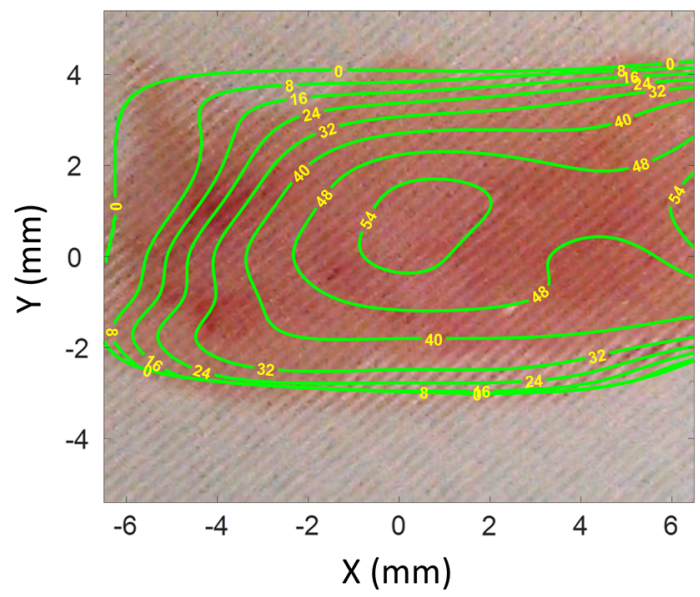

Fig. 9 (a) Photograph of 6-mm thick tonsil overlaid with contour lines labeling the time required to reach $63 \%$ of the maximum NBF concentration. (b) Photograph of 6-mm tonsil overlaid with contour lines labeling percent not diffused after $5 \mathrm{~h}$.

(a)

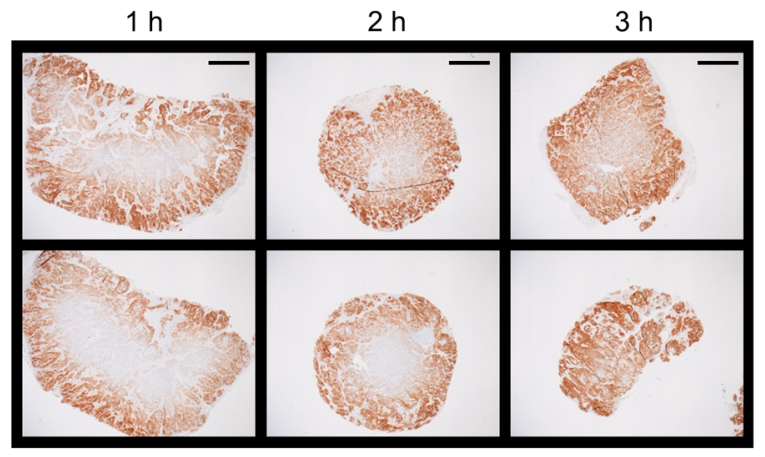

(b)

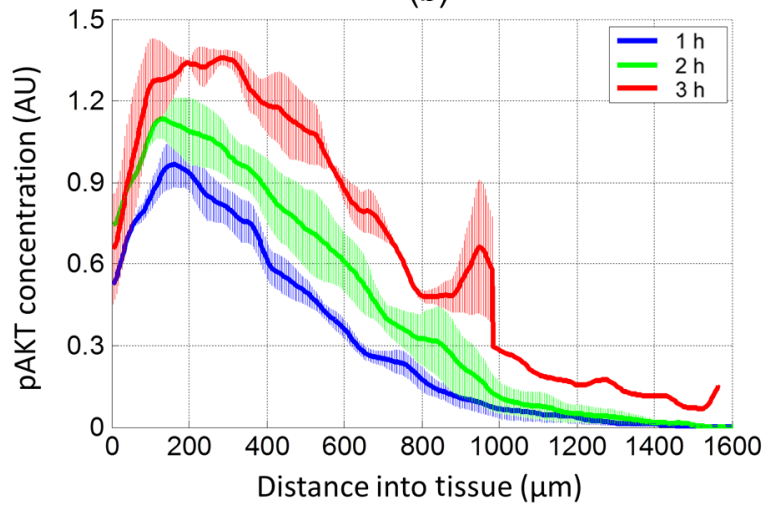

(c)

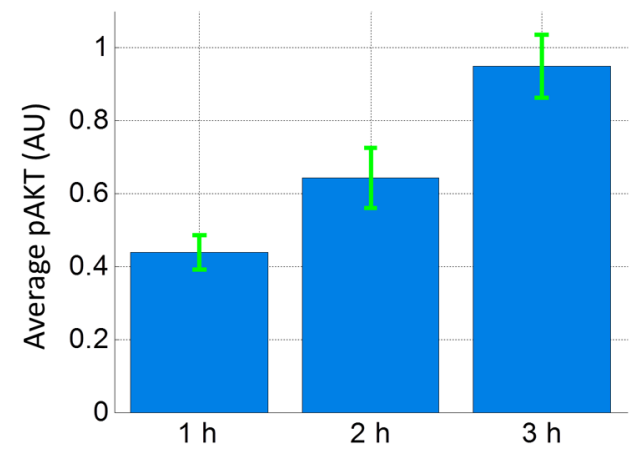

Fig. 10 (a) Amount of pAKT in 3- to 4-mm calu-3 xenografts submerged in formalin for $1 \mathrm{~h}$ (left), $2 \mathrm{~h}$ (middle), and $3 \mathrm{~h}$ (right). Scale bar $=1 \mathrm{~mm}$. (b) Concentration of pAKT versus distance into tissue from nearest edge. (c) Average pAKT expression for tissue samples submerged in $10 \%$ formalin for 1,2 , and $3 \mathrm{~h}$. Error bar width represents the standard deviation. Quantitative PAKT levels relative to common gold standard.

protein. This result would indicate that pAKT preservation is indeed largely dependent on the localized concentration of formaldehyde in the tissue.

\section{Discussion}

Previous researchers used US technology to interrogate tissue specimens before and after days to weeks of formalin fixation. ${ }^{25,29}$ However, these techniques lacked the ability to distinguish the effects of cross-linking and reagent diffusion and had coarse temporal resolution, which prevented measuring the rate of these processes. In the present work, tissue samples were placed in cold formalin to suppress cross-linking, thus enabling formaldehyde molecules to diffuse throughout the tissue before cross-link formation was completed with heat. Due to the distinct sound velocities of formalin and free fluid within the 
tissue, as the cross-linking agent penetrated the tissue, the overall transit time changed relative to the fixative's concentration, generating tens of nanoseconds of acoustic phase retardation. This small time interval was resolved with a developed TOF calculation algorithm capable of resolving temporal differentials on the order of hundreds of picoseconds. The measurement was sensitive and fast enough to actively monitor diffusion in real time. The change in TOF through tissue regularly increased when the tissue was incubated in a solution with a slower speed of sound, and conversely decreased when the tissue was incubated in a solution with a faster acoustic velocity. The reversibility of the TOF's amplitude and polarity in response to different formalin concentrations was consistent with diffusion being the primary phenomenon observed and demonstrated the signal was not significantly impacted by cross-link formation. Additionally, tissue sample decay amplitudes, decay rates, and polarities were all consistent with expectations from Eq. (1) and Fick's law. With this in mind, we conclude that the TOF signal was primarily, if not completely, derived from passive diffusion and the resultant change to the tissue's cumulative speed of sound and not a secondary effect such as tissue expansion/contraction or cross-linking activity.

With this acoustic TOF technology, one can now study and quantify the spatially and temporally varying diffusion process in tissues undergoing fixation. Active monitoring of fixative concentration could be used to ensure the presence of sufficient fixative throughout a sample despite large disparities between tissue types, tissue heterogeneity, or intersample variability. In particular, one might expect significant deviation in diffusion rates between different types of tissues due to their unique properties and compositions. Additionally, because TOF is an integrated signal, this approach could be used to quantify diffusion into a sample composed of multiple distinct tissue types. This technique could be instrumental to standardizing and optimizing tissue fixation by ensuring all tissues receive the optimal amount of cross-linking agent and documenting the preanalytical processes each sample was subjected to. Furthermore, it could be critical to the preservation of certain labile biomarkers that are highly sensitive to the local fixative concentration in the tissue. As an example, the preservation of pAKT was shown to be highly dependent on diffusion time. Verification of adequate fixative across all areas of a clinical tissue sample with this technique would enable a clinician to interpret a loss of pAKT staining as a true biologic phenomenon rather than a preanalytical artifact. In future work, we will therefore study the diffusion rates of formaldehyde in other clinically and biologically relevant tissues, as well as the TOF characteristics of other steps in tissue processing protocols, to better understand which diagnostic assays will most benefit from standardized and quantifiable tissue fixation.

\section{References}

1. M. Werner et al., "Effect of formalin tissue fixation and processing on immunohistochemistry," Am. J. Surg. Pathol. 24, 1016-1019 (2000).

2. R. Thavarajah et al., "Chemical and physical basics of routine formaldehyde fixation," J Oral Maxillofac. Pathol. 16, 400-405 (2012).

3. M. Titford, "Formalin fixation," Arch. Pathol. Lab. Med. 136, 137 (2012); author reply $137-138$.

4. A. C. Wolff et al., "American Society of Clinical Oncology/College of American Pathologists guideline recommendations for human epidermal growth factor receptor 2 testing in breast cancer," J. Clin. Oncol. 25, 118-145 (2006).
5. M. Plebani, "Errors in clinical laboratories or errors in laboratory medicine?," Clin. Chem. Lab. Med. 44, 750-759 (2015).

6. P. Bonini et al., "Errors in laboratory medicine," Clin. Chem. 48, 691698 (2002).

7. A. R. Morales et al., "Continuous-specimen-flow, high-throughput, 1hour tissue processing: system for rapid diagnostic tissue preparation," Arch. Pathol. Lab. Med. 126, 583 (2002).

8. C. D. Ainley and J. W. Ironside, "Microwave technology in diagnostic neuropathology," J. Neurosci. Methods 55, 183-190 (1994).

9. L. Antunes et al., "Possible role of tissue shrinkage in high-temperature antigen retrieval," Histopathology 48, 471-473 (2006).

10. R. Paulraj and J. Behari, "Single strand DNA breaks in rat brain cells exposed to microwave radiation," Mutat. Res./Fundam. Mol. Mech. Mutagen. 596, 76-80 (2006).

11. A. Yabuki et al., "The effects of microwave-irradiated fixation for postmortem changes of the kidney," J. Vet. Med. Sci. 66, 705-707 (2004).

12. D. Chafin et al., "Rapid two-temperature formalin fixation," PLoS One 8, e54138 (2013).

13. J. Winkelman, "Kinetics and chemical equilibrium of the hydration of formaldehyde," Chem. Eng. Sci. 57, 4067-4076 (2002).

14. C. Cinti et al., "Activated AKT as an indicator of prognosis in gastric cancer," Virchows Arch. 453, 449-455 (2008).

15. J. I. Kreisberg et al., "Phosphorylation of AKT $\left(\operatorname{Ser}^{473}\right)$ is an excellent predictor of poor clinical outcome in prostate cancer," Cancer Res. 64, 5232-5236 (2004).

16. F. Meric-Bernstam et al., "Influence of biospecimen variables on proteomic biomarkers in breast cancer," Clin. Cancer Res. 20, 3870 (2014).

17. C. Bonnas et al., "Effects of cold ischemia and inflammatory tumor microenvironment on detection of PI3K/AKT and MAPK pathway activation patterns in clinical cancer samples," Int. J. Cancer 131, 16211632 (2012).

18. A. P. Theiss et al., "Immunohistochemistry of colorectal cancer biomarker phosphorylation requires controlled tissue fixation," PLoS One 9, e113608 (2014).

19. N. S. Goldstein et al., "Minimum formalin fixation time for consistent estrogen receptor immunohistochemical staining of invasive breast carcinoma," Am. J. Clin. Pathol. 120, 86-92 (2003).

20. N. Arima et al., "The importance of tissue handling of surgically removed breast cancer for an accurate assessment of the Ki-67 index," J. Clin. Pathol., 1-5 (2015).

21. S. Kalkman, M. W. Barentsz, and P. J. van Diest, "The effects of under 6 hours of formalin fixation on hormone receptor and HER2 expression in invasive breast cancer," Am. J. Clin. Pathol. 142, 16-22 (2014).

22. C. Beaulieu, "The basis of anisotropic water diffusion in the nervous system - a technical review," NMR Biomed. 15, 435-455 (2002).

23. C. H. Fox et al., "Formaldehyde fixation," J. Histochem. Cytochem. 33, 845-853 (1985).

24. K. G. Helander, "Kinetic studies of formaldehyde binding in tissue," Biotech. Histochem. 69, 177-179 (1994).

25. J. C. Bamber et al., "Ultrasonic propagation through fixed and unfixed tissues," Ultrasound Med. Biol. 5, 159-165 (1979).

26. D. R. Bauer et al., "Dynamic subnanosecond time-of-flight detection for ultra-precise diffusion monitoring and optimization of biomarker preservation," Proc. SPIE 9040, 90400B (2014).

27. M. Vassilakopoulou et al., "Preanalytical variables and phosphoepitope expression in FFPE tissue: quantitative epitope assessment after variable cold ischemic time," Lab. Invest. 95, 334-341 (2014).

28. S. Gündisch et al., "Critical roles of specimen type and temperature before and during fixation in the detection of phosphoproteins in breast cancer tissues," Lab. Invest. 95, 561-571 (2015).

29. J. C. Bamber, C. R. Hill, and J. A. King, "Acoustic properties of normal and cancerous human liver-II. Dependence of tissue structure," Ultrasound Med. Biol. 7, 135-144 (1981).

Daniel R. Bauer is a scientist at Roche Tissue Diagnostics, where he researches methods of preserving and quantitating labile cancer biomarkers. He received his BS, MS, and PhD degrees from the College of Optical Sciences at the University of Arizona, where he studied multimodality biomedical imaging for improved cancer detection.

Biographies for the other authors are not available. 